\title{
Xenon GPSC high-pressure operation with large-area avalanche photodiode readout
}

\author{
L.C.C. Coelho ${ }^{\text {a }}$, J.A.M. Lopes ${ }^{\mathrm{a}, \mathrm{b}, *}$, D.S. Covita ${ }^{\mathrm{a}}$, A.S. Conceição ${ }^{\mathrm{a}}$, J.M.F. dos Santos ${ }^{\mathrm{a}}$ \\ ${ }^{a}$ Departamento de Física da Universidade de Coimbra, 3004-516 Coimbra, Portugal \\ ${ }^{\mathrm{b}}$ Instituto Superior de Engenharia de Coimbra, 3030-199 Coimbra, Portugal
}

Received 16 December 2006; accepted 27 February 2007

Available online 13 March 2007

\begin{abstract}
The performance of a xenon high-pressure gas proportional scintillation counter (GPSC) instrumented with a large area avalanche photodiode (LAAPD) as the VUV-photosensor has been investigated for filling pressures from 1 up to 10 bar, for $22-$ and 60 -keV photons. The LAAPD photosensor is placed directly within the xenon envelope, as a substitute for the photomultiplier tube, avoiding the constraints of the use of a quartz scintillation window for GPSC-photosensor coupling, which absorbs a significant amount of scintillation and is a drawback for applications where large detection areas and high filling pressures are needed. The lowest energy resolutions are achieved for pressures around 5 bar $(4.5 \%$ and $3.0 \%$ full width at half-maximum (FWHM), for 22- and $60-\mathrm{keV}$ photons, respectively). Increasing the pressure to the 8 bar range, competitive energy resolutions of $5.0 \%$ and $3.6 \%$ are still achieved for $22-$ and $60-\mathrm{keV}$ photons, respectively. This detector could be a compelling alternative in applications where compactness, large detection area, insensitivity to strong magnetic fields, room temperature operation, large signal-to-noise ratio and good energy resolution are important requirements.
\end{abstract}

(C) 2007 Elsevier B.V. All rights reserved.

PACS: 29.30.Kv; 29.40.Cs; 29.40.Mc; 29.40.Wk

Keywords: Xenon; X-ray detection; Gas proportional counter; LAAPD

\section{Introduction}

High-pressure xenon gas detectors with large area detection capability are attractive for hard X-ray detection applications such as X-ray digital radiography, synchrotron radiation studies, crystallography, $\mathrm{X}$ - and $\gamma$-ray astronomy. In the last decade, micropattern electronavalanche multiplier systems such as microstrip plate and grid electron multiplier (GEM), which are faster and present a much higher count-rate capability, substituted for the often used multiwire plate chamber technology. Nevertheless, gas proportional scintillation counters (GPSCs) [1], based on the production of secondary

\footnotetext{
*Corresponding author. Departamento de Física da Universidade de Coimbra, 3004-516 Coimbra, Portugal. Tel.: + 351239410667 ; fax: +351239829158 .

E-mail address: fcjam@gian.fis.uc.pt (J.A.M. Lopes).
}

scintillation in the gas as signal amplification, present superior energy resolution, high signal-to-noise ratio and high counting rates with reduced space-charge effects, when compared to gaseous detectors based on electron avalanche amplification. The superior performance of GPSCs result from the very low statistical fluctuations introduced by the secondary amplification process, in opposition to the charge amplification processes [1]. In addition, gaseous detectors based on electron avalanche amplification present a strong reduction in charge gain, as the gas pressure increases, and have not been used for pressures above a few bar [2-6].

A drawback in the operation of standard GPSCs is the use of photomultiplier tubes (PMTs) as the photosensors for the readout of the VUV-scintillation light produced in the noble gas fillings. Although presenting suitable high gains and low dark currents, the PMT has low quantum efficiency and is a bulky, power-consuming, complex and 
fragile device. Moreover, the need for thick scintillation windows to separate the GPSC gas volume from the PMT is a drawback for the use of GPSCs in applications requiring high gas-filling pressures, such as high-energy $\mathrm{X}$-ray and/or low-energy $\gamma$-ray spectrometry [7-9].

In response to these limitations, we have investigated the use of a CsI-coated microstrip plate, as the GPSC photosensor, placed directly in the xenon envelope as a substitute for the PMT avoiding, this way, the PMT and the scintillation window constraints. Although the energy resolution achieved with these GPSC/MSGC hybrid detectors $(11 \%$ full-width at half-maximum (FWHM) at $6 \mathrm{keV}[10])$ are not as good as that of PMT-based GPSCs $(8 \%$ FWHM at $6 \mathrm{keV})$, the performance is better than other xenon detectors based only on electron avalanche amplification. High-pressure operation of the xenon hybrid detector still demonstrated competitive energy resolutions of $\sim 7 \%$ and $4.5 \%$ for $22-$ and $60-\mathrm{keV} \mathrm{X}$-rays, respectively, in the 4- to 6-bar range [11].

We have also investigated the use of VUV-sensitive large area avalanche photodiodes (LAAPDs) as an alternative for replacing the PMTs in GPSCs $[12,13]$. When compared to the PMT and to the CsI-photocathode, the LAAPD can present higher quantum efficiencies. For LAAPDs from Advanced Photonix Inc. (API), typically $\sim 1.05$ electronhole pairs are produced per $170 \mathrm{~nm}$ photon [14], the xenon secondary scintillation, while for PMTs and CsI this number is $\sim 0.15$ and $\sim 0.30$, respectively. In addition, the compactness, ruggedness, reduced power consumption, insensitivity to strong magnetic fields [15] and windowless operation of the LAAPD make it a suitable candidate for high-pressure gas scintillation readout.

The small active area of the LAAPD photosensor is a drawback for large area applications. However, this limitation can be overcome by using several LAAPDs, a solution that has the additional advantage of 2D-position readout capability.

In this work, we investigate the performance of a xenon high-pressure GPSC, instrumented with a LAAPD for scintillation readout and using a very shallow scintillation gap of $1.5 \mathrm{~mm}$. Detector relative amplitude and energy resolution are studied as a function of xenon-filling pressure, in the range of 2-10 bar and for X-ray energies of 6,22 and $60 \mathrm{keV}$. The small scintillation gap and highpressure fillings allow a more compact detector design with higher count-rate capability and/or extend the detection range to hard X-rays. Former studies with a GPSC/ LAAPD detector [16] were limited to filling pressures up to 5 bar, due to poor electrical insulation, and it was not clear if the energy resolution could improve above 5 bar and/or if the used getters could be efficient at higher pressures.

\section{Experimental setup}

The schematic of the GPSC used in this work is depicted in Fig. 1. The radiation window is maintained at negative high-voltage $-\mathrm{HV}_{0}$, while mesh $\mathrm{G} 1 \quad(80-\mu \mathrm{m}$ diameter

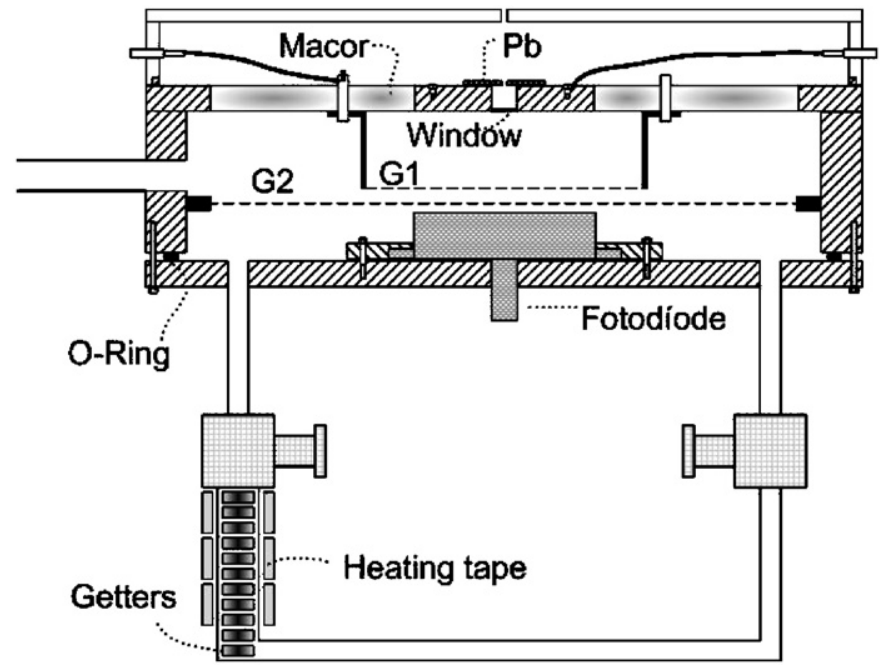

Fig. 1. Schematic of the present GPSC instrumented with a LAAPD photosensor substituting for the PMT.

stainless-steel wire with $900-\mu \mathrm{m}$ spacing) and its holder are kept at $-\mathrm{HV}_{1}$; mesh $\mathrm{G} 2$ and the detector body are grounded. The voltage difference between the detector window and G1 defines the electric field in the absorption region, which was kept just below the xenon scintillation threshold, $1 \mathrm{~V} \mathrm{~cm}^{-1} \mathrm{Torr}^{-1}$, throughout the work. The scintillation region is delimited by $\mathrm{G} 1$ and $\mathrm{G} 2$, being the electric field defined by $-\mathrm{HV}_{1}$. A Macor piece, glued with low vapour pressure epoxy to the detector body and to the radiation window holder is used for electrical insulation of the different electrodes. The holder of G1 is fixed to the Macor with screws, one of which is used as a feedthrough for the biasing voltage of G1. The radiation window is made of $50-\mu \mathrm{m}$ Kapton film, aluminised on the inner surface and epoxied to its holder electrode. The LAAPD VUV-photosensor is placed just below G2. The upper and the lower parts of the detector body and the LAAPD body are sealed with a Viton O-ring.

In this small GPSC prototype, the absorption/drift region and the scintillation region were designed to be shallow (1-cm deep and $1.5-\mathrm{mm}$ deep, respectively), in order to apply relatively low biasing-voltages, while keeping high-reduced electric fields (the electric field intensity divided by the gas pressure, $E / p)$.

The LAAPD is an API Deep-UV diode with a $16-\mathrm{mm}$ diameter active area [14]. The LAAPD is custom made and has several 3-mm diameter holes on its body to connect the outside to the inner chamber, which enclose the electrical contacts with the SHV socket, in order to have the same pressure in the opposite sides of the silicon wafer. Throughout this work, the maximum LAAPD bias was limited to a safe value of $1650 \mathrm{~V}$ to prevent any gas discharge between its electrodes, which would destroy the diode, since the gas inside the LAAPD is xenon and not dry nitrogen as in standard LAAPDs. Although this biasing voltage corresponds to a low photosensor gain ( 25 [14]), high performance is already achieved due to both high 
scintillation amplification in the GPSC and high conversion-efficiency of $\mathrm{Xe}$ scintillation into charge in the LAAPD [12].

The detector was filled at different xenon pressures, from atmospheric up to $10 \mathrm{bar}$ and the pressure was kept constant during each set of measurements. The gas inlet and outlet ports of the detector are interconnected through a U-shaped tube, being one of the arms filled with nonevaporable getter (SAES Getters, St 707/washer/833). The getter heating was done by temperature-controlled electric heating tapes placed on the outside of the tube arm, maintaining the operating temperature steady in the range of $100-250^{\circ} \mathrm{C}$. The filling gas was continuously purified, by convection through the getters.

The charge signals of the LAAPD were fed through a CANBERRA 2006 charge-to-voltage preamplifier (sensitivity of $235 \mathrm{mV} / 10^{6}$ ion pairs) and a TENNELEC TC 243 linear amplifier $(1-\mu$ s peaking time constant) to a 1024multichannel pulse-height analyser. For peak amplitude and energy-resolution measurements, pulse-height distributions were fitted to a Gaussian function superimposed on a linear background, from which the centroid and the FWHM were determined.

\section{Experimental results and discussion}

The shallow scintillation region, $1.5 \mathrm{~mm}$ instead of the $10 \mathrm{~mm}$ typically used at $1-2$ bar, presents advantages in the GPSC operation at high pressures, delivering shorter lightburst pulses and allowing the use of low biasing voltages for the same $E / p$ established in the scintillation region. Thus, shaping-time constants as low as $1 \mu$ s were used in the present case, without any degradation in the detector performance. We have already shown that, for GPSC operation at $\sim 1$ bar, scintillation depths as low as $5 \mathrm{~mm}$ deliver the same performance as obtained with $10 \mathrm{~mm}$ [17]. In addition, we have shown that, for the $E / p$ values used in the scintillation region, GPSC are much more immune to grid misalignments than detectors based on electron avalanche amplification [18].



Fig. 2. Detector relative amplitude as a function of $E / p$ in the scintillation region, for the studied xenon pressures and for 59.6-keV $\gamma$-rays. Error bars are smaller than symbols size.
In Fig. 2, we present the detector relative pulse amplitude as a function of $E / p$ in the scintillation region, using the 59.6-keV peak, and for different xenon-filling pressures in the 2- to 10-bar range. In all cases, the pulse amplitude exhibits the characteristic linear behaviour of the xenon electroluminescence, with a threshold at about $1 \mathrm{~V} / \mathrm{cm}^{1} /$ Torr $^{1}$. For each pressure, the maximum achieved $E / p$ was limited by electrical insulation and maintained below the onset of electric breakdown.

Figs. 3(a) and (b) depict the detector energy resolution, FWHM, as a function of $E / p$ in the scintillation region for 2-mm collimated beams of 22.1- and 59.6-keVphotons, respectively, and for the different gas-filling pressures. For low scintillation output, i.e. low $E / p$, the energy resolution of GPSCs depends strongly on the number of VUV photons produced in the scintillation region, presenting a fast decrease with increasing $E / p$. As the number of VUV photons increases this dependence becomes less significant and the energy resolution becomes constant, eventually degrading for larger biasing voltages. This is attributed to the onset of leak currents and microdischarges, which arise at high voltages. As for higher filling pressures the scintillation output is larger, its contribution to the energy resolution becomes negligible for lower values of $E / p$. This explains why the $E / p$ value, for which the lowest energy

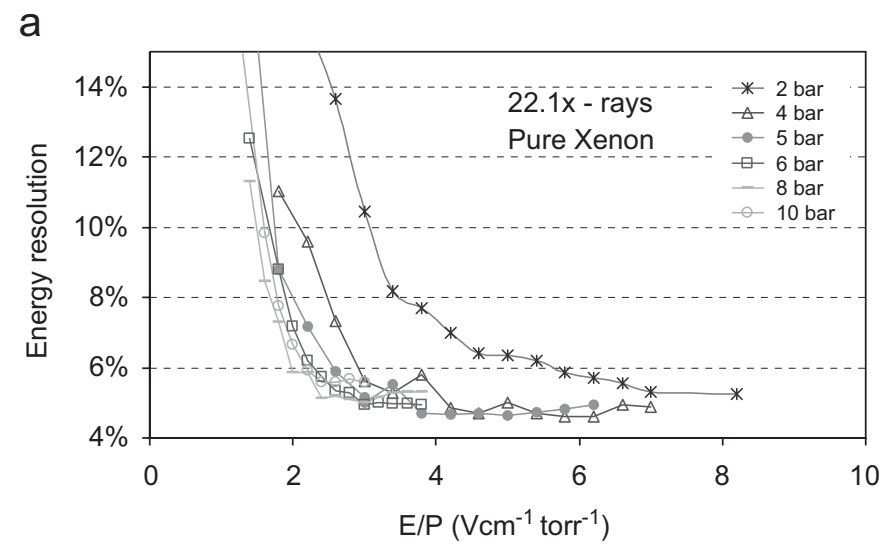

b

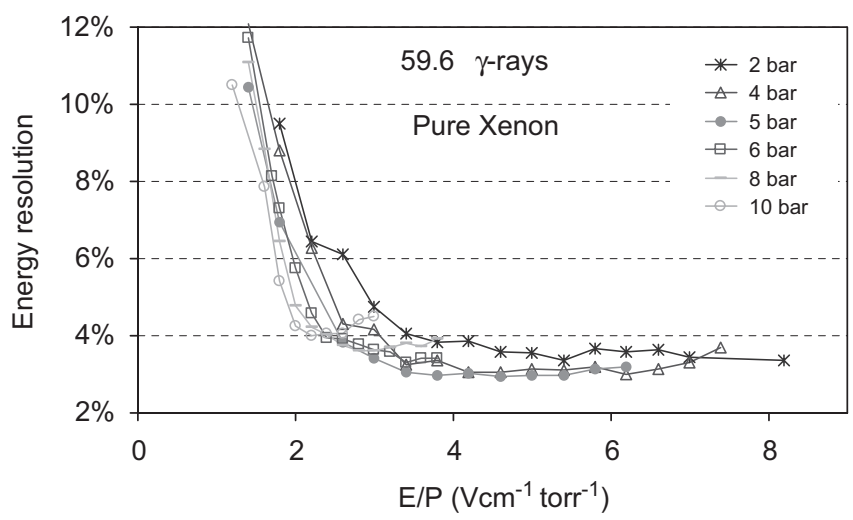

Fig. 3. Detector energy resolution for 22-keV X-rays (a) and 59.6-keV $\gamma$-rays (b) as a function of $E / p$ in the scintillation region, for the different pressures. Error bars are smaller than symbols size. 
resolution is achieved, decreases with the gas pressure, being around $9,6,4,3$ and $2.4 \mathrm{~V} / \mathrm{cm}^{1} /$ Torr $^{1}$ for $2,4,6,8$ and $10 \mathrm{bar}$, respectively. This trend, of lower energy resolutions for lower reduced electric fields, with increasing xenon pressure, is also present in electron avalanche proportional counters [19].

Fig. 4 presents the best-achieved detector energy resolution as a function of pressure, for 22- and $60-\mathrm{keV}$ photons. The shallow scintillation region results in reduced scintillation output for low energy X-rays at low pressures and, thus, in poor energy resolution. For $6 \mathrm{keV}$, good energy resolutions of $\sim 8 \%$ FWHM are only achieved for pressures above $5 \mathrm{bar}$, while for $22 \mathrm{keV}$ good energy resolutions are achieved for pressures around $4 \mathrm{bar}$, being the resolution significantly higher at 2 bar. The best-achieved energy resolutions are $4.5 \%$ and $3.0 \%$ FWHM for 22- and $60-\mathrm{keV}$ photons, respectively, and are obtained for pressures around 5 bar. However, competitive energy resolutions of $5.0 \%$ and $3.6 \%$ FWHM are still achieved at 8 bar. The energy resolutions achieved at high pressure are similar to those obtained with the GPSC of Ref. [9], which features a 7-cm deep drift region, a $10-15-\mathrm{mm}$ deep scintillation region and 1.2 bar xenon atmosphere. For standard Xe-GPSCs operated with PMTs, typical energy resolutions of $6.1 \%$ and $3.6 \%$ were achieved for $30-$ and $60-\mathrm{keV}$ photons, respectively, at $5.5 \mathrm{bar}$, while at 9 bar the resolution degrades to $7.6 \%$ and $3.6 \%$, respectively $[20,21]$. These energy resolutions are much better than those of electron avalanche detectors: at filling pressures of 1,5 and $10 \mathrm{bar}$, typical values obtained with PCs [19] are $7.7 \%, 8.3 \%$ and $9.6 \%$, and $5.5 \%, 6.0 \%$ and $6.5 \%$, for $22-$ and $60-\mathrm{keV}$ photons, respectively.

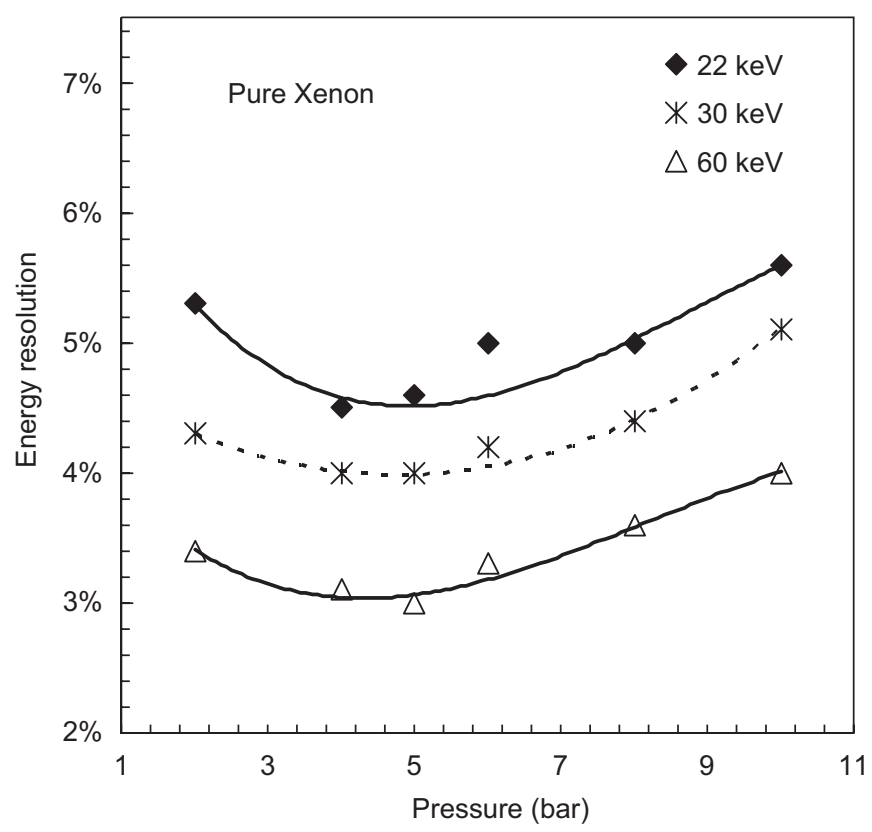

Fig. 4. Lowest detector energy resolution as a function of pressure for 22 , 30 and $60 \mathrm{keV}$. Error bars are smaller than symbols size. a
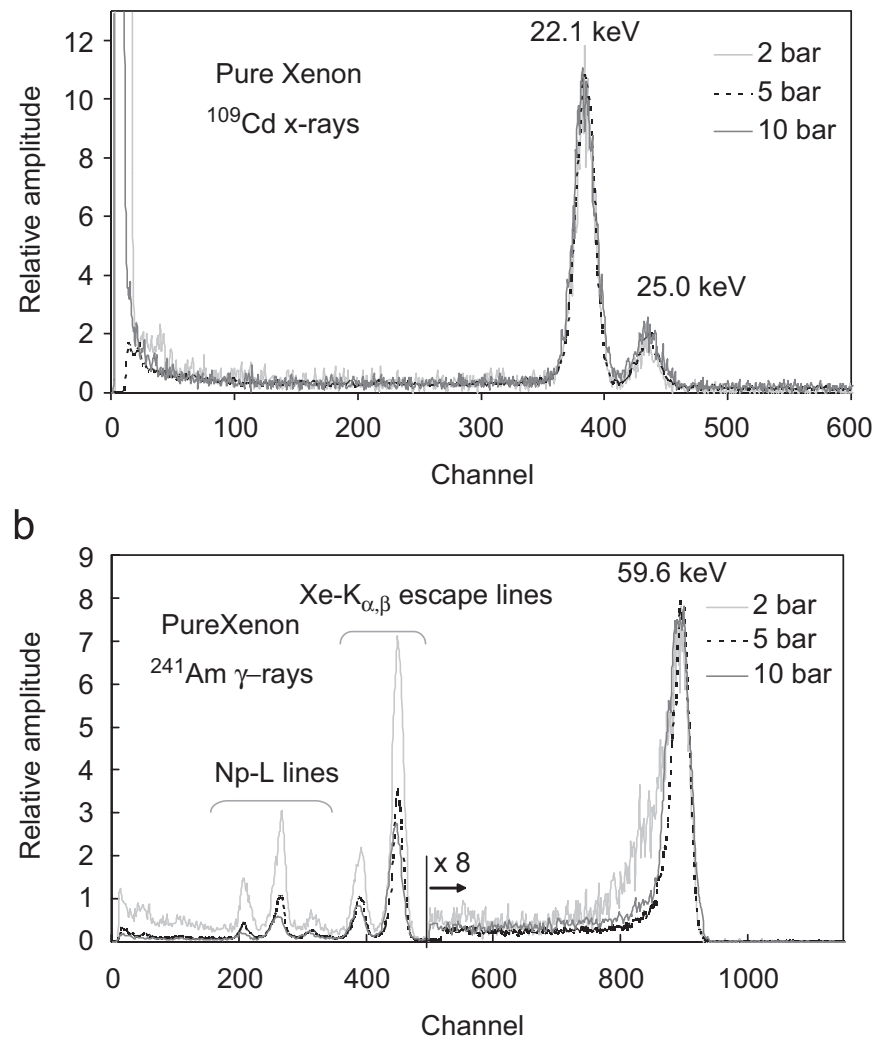

Fig. 5. Typical pulse-height distributions obtained with the detector for $\mathrm{X}$ - and $\gamma$-rays from ${ }^{109} \mathrm{Cd}$ (a) and ${ }^{241} \mathrm{Am}$ (b) radioactive sources, for different xenon-filling pressures.

Fig. 5 depicts typical detector pulse-height distributions obtained for ${ }^{109} \mathrm{Cd}$ (a) and ${ }^{241} \mathrm{Am}$ (b) radioactive sources for different gas pressures. Low plateau levels with good energy resolutions show the high potential of the xenon GPSC operating at high pressures. We attribute the detector energy resolution degradation at high pressures to the fact that best energy resolutions are achieved for lower reduced electric fields, for which the xenon scintillation efficiency is significantly lower, resulting in increased scintillation-process statistical fluctuations.

\section{Conclusions}

We have investigated the performance characteristics of a high-pressure xenon GPSC instrumented with a LAAPD photosensor for scintillation readout. The performance was studied for the detection of $22-$ and $60-\mathrm{keV}$ photons and filling pressures ranging from 2 to $10 \mathrm{bar}$. High-pressure gas fillings extend the detection range to hard X-rays at the cost of small detector performance degradation.

We have used a shallow scintillation region, $1.5-\mathrm{mm}$ deep. We have shown that this gap is enough at high pressures, even for the detection of X-ray energies as low as $6 \mathrm{keV}$. The small scintillation gap allows a more compact detector design and higher count rate. 
Table 1

Operating conditions for the lowest energy resolutions achieved with the present detector

\begin{tabular}{lllll}
\hline $\begin{array}{l}\text { Pressure } \\
\text { (bar) }\end{array}$ & $\begin{array}{l}\text { Scintillation } \\
\text { region, } E / p \\
\left(\mathrm{~V} / \mathrm{cm}^{1} / \mathrm{Torr}^{1}\right)\end{array}$ & \multicolumn{2}{l}{ Energy resolution $(\%$ FWHM) } \\
\cline { 3 - 5 } & 8.2 & 5.3 & 4.3 & $60 \mathrm{keV}$ \\
\hline 2 & 6 & 4.5 & 4.0 & 3.4 \\
4 & 5 & 4.6 & 4.0 & 3.1 \\
5 & 4 & 5.0 & 4.2 & 3.0 \\
6 & 3 & 5.0 & 4.4 & 3.6 \\
8 & 2.4 & 5.6 & 5.1 & 4.0 \\
10 & & & & \\
\hline
\end{tabular}

Table 1 summarises the operating conditions and performance for which the lowest energy resolutions are achieved, with the present detector. The obtained energy resolutions are much better than those of electron avalanche detectors, and similar or slightly better than those obtained with standard GPSCs instrumented with PMTs. Best energy resolutions are achieved for pressures around 5 bar, presenting a small degradation with pressure for higher pressures, but competitive energy resolutions are still achieved for 8 and 10 bar.

\section{Acknowledgements}

This work was carried out at the Instrumentation Centre of the Physics Department of the University of Coimbra, and it was supported by project POCI/FIS/60534/2004 through POCI 2010 and FCT (Lisbon) programs.

\section{References}

[1] J.M.F. dos Santos, J.A.M. Lopes, J.F.C.A. Veloso, et al., X-ray Spectrom. 30 (2001) 373;

J.F.C.A. Veloso, J.M.F. dos Santos, C.A.N. Conde, Nucl. Instr. and Meth. A 457 (2001) 253.
[2] J. Nickles, H. Bräuning, A. Bräuning-Demian, et al., IEEE Trans. Nucl. Sci. NS-49 (2002) 808.

[3] A. Bondar, A. Buzulutskov, F. Sauli, L. Sheckhtman, Nucl. Instr. and Meth. A 419 (1998) 418.

[4] A. Bondar, A. Buzulutskov, L. Schekhtmann, Nucl. Instr. and Meth. A 481 (2002) 200.

[5] B.D. Ramsey, J.A. Apple, R.A. Austin, et al., Nucl. Instr. and Meth A 383 (1996) 424.

[6] M. Li, M.S. Dixit, P.C. Johns, Nucl. Instr. and Meth A 471 (2001) 215.

[7] G. Fazio, S. Giarusso, G. Manzo, et al., Nuo. Cim. C- Geo. and Spa. Phys. 20 (1997) 819.

[8] G.V. Volchenko, Y.M. Gavrilyuk, V.V. Kuz'minov, et al., Instrum. Exp. Techn. 42 (2001) 31.

[9] H. Natal da Luz, J.F.C.A. Veloso, J.M.F. dos Santos, et al., IEEE Trans. Nucl. Sci. NS-49 (2002) 2488.

[10] D.S.A.P. Freitas, J.F.C.A. Veloso, C.M.B. Monteiro, J.M.F. dos Santos, C.A.N. Conde, Nucl. Instr. and Meth. A 505 (2003) 228.

[11] L.C.C. Coelho, J.F.C.A. Veloso, D.S. Covita, L.F. Requicha Ferreira, J.M.F. dos Santos, Nucl. Instr. and Meth. A 569 (2006) 796.

[12] J.A.M. Lopes, J.M.F. dos Santos, R.E. Morgado, C.A.N. Conde, IEEE Trans. Nucl. Sci. NS-48 (2001) 312.

[13] C.M.B. Monteiro, J.A.M. Lopes, P.C.P.S. Simões, J.M.F. dos Santos, C.A.N. Conde, IEEE Trans. Nucl. Sci. NS-48 (2001) 1081.

[14] Deep UV 500 Windowless Séries LAAPD, Advanced Photonics Inc., Camarillo, CA.

[15] L.M.P. Fernandes, A. Antognini, M. Boucher, et al., Nucl. Instr. and Meth. A 498 (2003) 362.

[16] D.S. Covita, J.A.M. Lopes, M. Szawlowski, J.M.F. dos Santos, IEEE Trans. Nucl. Sci. NS-51 (2004) 1492.

[17] F.I.G.M. Borges, J.M.F. dos Santos, T.H.V.T. Dias, et al., Nucl. Instr. and Meth. A 422 (1999) 321.

[18] A.C.S.M. Bento, J.M.F. dos Santos, R.E. Morgado, IEEE Trans. Nucl. Sci. NS-44 (1997) 527.

[19] H. Sakurai, B.D. Ramsey, M.C. Weisskpf, Nucl. Instr. and Meth A 307 (1991) 504.

[20] A. Bolozdynya, V. Egorov, A. Koutchenkov, G. Safronov, G. Smirnov, S. Medved, V. Morgunov, Nucl. Instr. and Meth A 385 (1997) 225.

[21] A. Bolozdynya, V. Egorov, A. Koutchenkov, G. Safronov, G. Smimov, S. Medved, V. Morgunov, IEEE Trans. Nucl. Sci. NS-44 (1997) 1046 (and references therein). 